\title{
Doppler Effect of Nonlinear Waves and Superspirals in Oscillatory Media
}

\author{
Lutz Brusch, ${ }^{1}$ Alessandro Torcini, ${ }^{2}$ and Markus Bär ${ }^{1}$ \\ ${ }^{1}$ MPI for Physics of Complex Systems, Nöthnitzer Str. 38, D-01187 Dresden, Germany ${ }^{*}$ \\ ${ }^{2}$ Istituto Nazionale di Ottica Applicata, L.go E. Fermi 6, I-50125 Firenze, Ital
}

(Dated: August 13, 2018)

\begin{abstract}
Nonlinear waves emitted from a moving source are studied. A meandering spiral in a reactiondiffusion medium provides an example, where waves originate from a source exhibiting a back-andforth movement in radial direction. The periodic motion of the source induces a Doppler effect that causes a modulation in wavelength and amplitude of the waves ("superspiral"). Using the complex Ginzburg-Landau equation, we show that waves subject to a convective Eckhaus instability can exhibit monotonous growth or decay as well as saturation of these modulations away from the source depending on the perturbation frequency. Our findings allow a consistent interpretation of recent experimental observations concerning superspirals and their decay to spatio-temporal chaos.
\end{abstract}

PACS numbers: 82.40.Ck, 05.45.-a, 47.54.+r

Introduction. - Periodic nonlinear waves are a trademark of nonequilibrium systems [1]. In one dimension (1D), they can appear in systems with periodic boundary conditions (BCs) as well as in open geometries, where BCs select a unique pattern 22. In two dimensions (2D), rotating spiral waves are frequently observed. Therein, periodic waves emerge from the region of the spiral tip (core) and propagate in radial direction. The aim of this paper is to investigate the effects of perturbing sources of nonlinear waves and their implications for the dynamics of spiral waves. We employ the complex GinzburgLandau equation (CGLE), which provides an universal description of spatially extended oscillatory systems near a supercritical Hopf bifurcation [3, 4].

In this framework, we perturb a source of periodic waves in 1D by moving its position back-and-forth in space. This motion of the source leads to a modulation in amplitude, wavelength and frequency of the emitted waves. We find that the modulation of the nonlinear waves is uniquely determined by the temporal period of the source motion in contrast to linear waves emitted by a moving source, where the source velocity is the relevant quantity. The richest scenario is found if the emitted waves are convectively Eckhaus unstable. If we consider a periodically moving source in the latter case, the modulations in amplitude and period of the waves may (a) get exponentially damped, (b) saturate or (c) grow monotonously far away from the source depending on the frequency of the applied forcing.

Such a periodically moving source reproduces the radial dynamics of rotating spiral waves subject to external forcing 5$]$ or to the frequently observed oscillatory instability termed "meandering" [6]. In $2 \mathrm{D}$, the radial modulations caused by meandering lead to a second spiral superimposed on the simple rotating spiral; the resulting structure is called superspiral (see Fig. 1). Our study provides insight in the nonlinear behavior of such superspirals extending earlier work based on a linear analysis [7]. Unbounded growth of the modulation will lead to occurrence of space-time defects in 1D and superspiral breakup in $2 \mathrm{D}$, provided the system is sufficiently large.

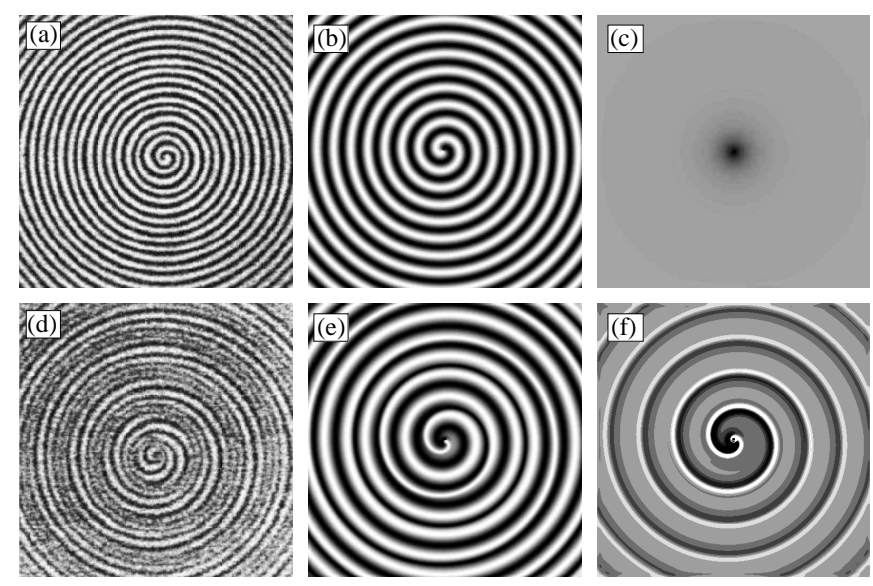

FIG. 1: Simple spiral waves (a-c) and superspirals (d-f) are observed in experiments (a,d) of the BZ system [8] and in numerical simulations of the CGLE (1). (b,e) show $\operatorname{Re}[A]$ and $(\mathrm{c}, \mathrm{f})|A|$. (b,c) $\mu=1$ and $(\mathrm{e}, \mathrm{f})$ inhomogeneous $\mu=$ $1+0.7 \exp \left(-r^{2} / 10\right)$. Other parameters are $c_{1}=3.5, c_{3}=0.34$, system size is $512 \times 512$.

Experimental evidence of such behavior was found in the Belousov-Zhabotinsky (BZ) reaction [8]. The first experimental observation of a superspiral was reported for a spiral subject to strong external forcing near its core [9].

Complex Ginzburg-Landau equation. - Consider a spatially extended oscillatory medium described by the complex Ginzburg-Landau equation [1, 4]

$$
\partial_{t} A=\mu A+\left(1+i c_{1}\right) \Delta A-\left(1-i c_{3}\right)|A|^{2} A
$$

with $\mu=1$. The complex field $A(r, t)$ gives amplitude and phase of local oscillations depending on real coefficients $c_{1}, c_{3}$ determined by the underlying specific model at the onset of oscillations. Eq. (11) exhibits plane wave solutions $A(r, t)=\sqrt{1-q^{2}} \mathrm{e}^{i(q r-\omega t)}$ with $\omega=-c_{3}+q^{2}\left(c_{1}+c_{3}\right)$ in an infinite or periodic 1D medium with $\Delta=\partial_{r}^{2}$. For fixed control parameters, these waves become unstable if the wavenumber $q$ is bigger than the 


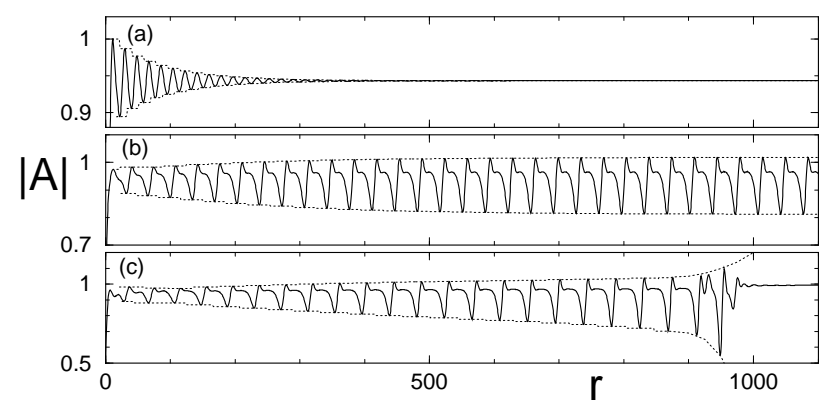

FIG. 2: Doppler effect of nonlinear waves due to periodic oscillations of the source at the left boundary. Eq. (1) was integrated for increasing forcing periods (a) $\tau=8$, (b) $\tau=13$, (c) $\tau=15$ with Eq. (5) and $c_{1}=3.5, c_{3}=0.4, R_{S}=1$. Dotted lines are guides to the eye that converge to $|A|_{\text {min }}$ and $|A|_{\max }$ in (b).

Eckhaus wavenumber $q_{E}^{2}=\left(1-c_{1} c_{3}\right) /\left(2\left(1+c_{3}^{2}\right)+1-c_{1} c_{3}\right)$. The plane waves represent a one-parameter family that is parametrized by the wavenumber $q$. A source of periodic waves at $r=0$ is easily realized by applying the BCs

$$
A(r=0)=0 \quad \text { and } \quad \partial_{r} A(r=L)=0 .
$$

The specification of these BCs leads to the selection of a unique wavenumber $q_{S}$. In this case, the exact solution of the CGLE is of the form

$$
A(r, t)=F(r) \mathrm{e}^{i(f(r)-\omega t),}
$$

with the asymptotic behavior $d f(r) / d r \rightarrow q_{S}, F(r) \rightarrow$ $\sqrt{1-q_{S}^{2}}$ for $r \rightarrow \infty$ (far-field) and $d f(r) / d r \sim r, F(r) \sim$ $r$ for $r \rightarrow 0$ (near-field). An analytic expression for $q_{S}$ has been derived [2, 10]

$$
0=\left(c_{1}+c_{3}\right) q_{S}^{2}+3 \alpha\left(c_{1}, c_{3}\right) q_{S}-c_{3}-2 c_{1} \alpha\left(c_{1}, c_{3}\right)^{2},
$$

where $\alpha$ is a function of the control parameters $c_{1}, c_{3}$ [11].

In 2D, Eq. (1) with $\Delta=\partial_{r}^{2}+1 / r \partial_{r}+1 / r^{2} \partial_{\theta}^{2}$ possesses rotating spiral solutions with $A=0$ in the spiral core and in the far-field a selected wavenumber $q_{S}$, which is similar to (4) as verified numerically [2, 10]. Eq. (4) allows to discriminate if the source or the spiral core emits stable (Eckhaus unstable) wavetrains with $q_{S}<q_{E}\left(q_{S}>q_{E}\right)$. Further analysis showed that the Eckhaus instability is of convective nature and becomes absolute for sufficiently large values of $c_{1}$ and $c_{3}$ [12]. At given parameters, we may define a wavenumber $q_{A}>q_{E}$ that characterizes absolutely unstable waves with $q>q_{A}$. In finite systems, spirals were found to be stable as long as $q_{S}<q_{A}[12$, 13].

Simulation results. - Here, we analyze the effects of an instability or of an external perturbation at the source or at the spiral core (meandering). We choose to perturb the source in Eq. (2) by varying its position $r_{S}$. Consequently,

$$
\begin{array}{r}
A\left(r \leq r_{S}\right)=0 \quad \text { with } \quad r_{S}=R_{S} \cos (2 \pi t / \tau) \\
\text { and } \quad \partial_{r} A(r=L)=0,
\end{array}
$$

is used as BC of Eq. (11) in 1D. The core motion of a meandering spiral can be viewed as a source moving along a circle in $2 \mathrm{D}$. A projection onto a radial direction for fixed angle yields a periodic back-and-forth motion. A sinusoidal motion like in Eq. (5) is expected near onset, where the normal form of meandering [14] provides a valid description.

Hence, the BC in Eq. (5) also captures the radial dynamics of a meandering spiral. In the numerical simulations the moving source initially modulates the local wavenumber which subsequently leads to a modulation of the wave amplitude. For large $r$ (far-field), we observe modulations with a period $T$ that is equal to the forcing period $\tau$ and independent from $R_{S}$. First, we forced sources (respectively "spirals") in the parameter region where $q_{S}<q_{E}$, in this case the perturbation is always damped out while the waves move away from the source. A larger variety of responses occurs in the convectively Eckhaus unstable regime, where the unperturbed source selects a wavenumber $q_{S}$ in the interval $\left[q_{E}, q_{A}\right]$. Fig. 2 shows three qualitatively different resulting profiles of the amplitude $|A|$ as a function of the radial coordinate. Figs. 2(a), (b) and (c) correspond to increasing values of the forcing period $\tau$. For small $\tau$-values, the result is similar to the case with $q_{S}<q_{E}$, the amplitude modulation is exponentially damped and is barely visible at sufficiently large $r$ (Fig. 2(a)). For intermediate $\tau$, the amplitude modulation first grows and then reaches saturation (Fig. $2(\mathrm{~b})$ ). The profile of $|A|$ is periodic and travels with a non-zero velocity; in the far-field, the radial dynamics resemble a so-called modulated amplitude wave (see below). Finally for large $\tau$, the amplitude modulation grows monotonically with $r$ and space-time defects are formed at the breakup radius $r=R_{B U} \approx 950$ (Fig. 2(c)). For simulations with $q_{S}>q_{A}$, we always observe monotonous growth of the modulation leading to space-time defects.

A drawback of the homogeneous CGLE in 2D is that it does not support meandering spirals. A non-saturating meandering instability has been observed for large values of $c_{1}$ [15. Alternatively, addition of a heterogeneity near the spiral core leads to meandering behavior similar to the one typically seen in reaction-diffusion systems [16]. Figs. 1 (e),(f) show such a superspiral. The corresponding quantities for a regular spiral are shown in Fig. 1 (b),(c). As in Fig. 2(b) and in the recent experiment by Zhou and Ouyang (see Fig. 1(d) and [8]) the amplitude modulation shown in Fig. 1(f) saturates in the far-field. In the following, the study will be limited to the radial dynamics. One of the new results of our analysis is that the modulation of the amplitude may eventually saturate (see Fig. 2(b)). It also travels with constant shape and with a speed different from that of the underlying phase waves. To summarize, periodically moving sources emit modulated amplitude waves analogous to the way stationary sources send out plane waves.

Modulated amplitude waves (MAWs) and superspirals. - MAWs are solutions of the CGLE and have the follow- 


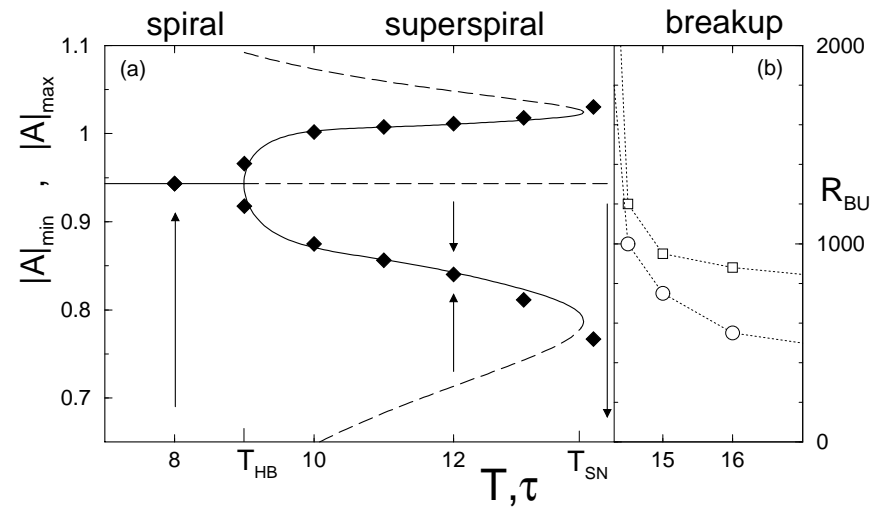

FIG. 3: (a) Bifurcation diagram for MAWs with period $T$ and existence domains of spirals, superspirals and spiral breakup for various forcing periods $\tau$. Curves were computed as in [17, 18] and symbols denote minima and maxima of $|A|$ as measured in simulations, parameters are the same as in Fig. 2. Solid (dashed) curves correspond to stable (unstable) solutions. Arrows (same for $|A|_{\max }$ ) indicate the evolution of initial perturbations as they move away from the source. Their asymptotic values depend on $T=\tau$ but not on $R_{S}$. (b) Breakup radius $R_{B U}$ at which defects firstly occur in simulations. Smaller remaining spirals result from larger $R_{S}$ ( $R_{S}=5$ circles, $R_{S}=1$ squares $)$.

ing general form

$$
A(r, t)=a(z) e^{i \phi(z)} e^{i(q r-\omega t)},
$$

with unknown periodic functions $a$ and $\phi$ of the comoving coordinate $z=r-v t$ [17]. The analysis of MAWs has revealed that they bifurcate from plane waves with $q>q_{E}$. MAWs form a two-parameter family of waves described by the wavelength $P$ of the modulation of the amplitude $a=|A|$ and by the spatially averaged phase gradient $\nu=q+\left\langle\partial_{r} \phi\right\rangle$. The velocity $v$ and the period $T=P / v$ can be derived from these quantities. The average phase gradient is conserved as long as no space-time defect is formed. For periodically perturbed sources, the phase gradient value is fixed to $\nu \equiv q_{S}$. Therefore, only one free parameter is left. For the present purpose it is most convenient to use the period $T$. The resulting bifurcation diagram is shown in Fig. 3 for the case $q_{S}>q_{E}$ (Eckhaus unstable range). In the Eckhaus stable regime, no stable MAWs exist.

Fig. 3 reveals that stable MAWs exist for periods $T$ in the interval $\left[T_{H B}, T_{S N}\right]$. They are "born" in a Hopf bifurcation (HB) and "die" in a saddle-node bifurcation (SN). For $T<T_{H B}$, the plane wave is stable and for $T>T_{S N}$ no MAWs exists and the dynamics leads to the formation of defects 17, 19. This clarifies our findings in Fig. 2: the profiles in Fig. 2(a), 2(b) and 2(c) correspond to forcing within the regions $\tau<T_{H B}, T_{H B}<\tau<T_{S N}$ and $T_{S N}<\tau$, respectively. Fig. 3 (b) shows the breakup radius $R_{B U}$ found in simulations for periods $\tau>T_{S N}$. It is crucial to realize that the properties of the sat-

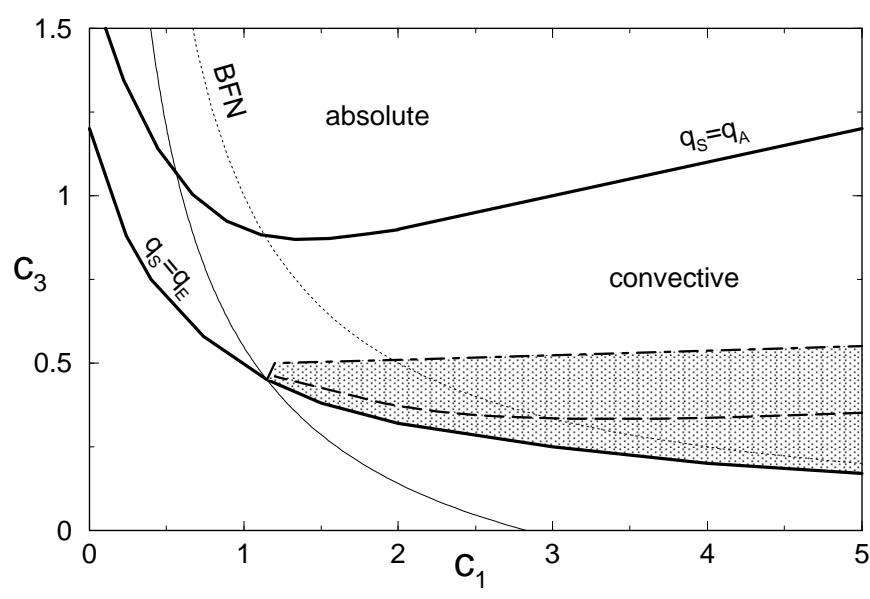

FIG. 4: Phase diagram indicating the region (shaded) where superspirals may occur in the CGLE for "rocking" sources. Simple spirals with selected $q_{S}\left(c_{1}, c_{3}\right)$ are convectively unstable between the thick solid curves. Superspiral breakup is only possible between the dashed and dash-dotted curves. The Eckhaus instability for arbitrary $q$ is supercritical above the thin solid curve and the thin dotted curve indicates the Benjamin-Feir-Newell line (BFN).

urated modulations, observed in numerical simulations with a periodically moving source, indeed correspond to the MAWs with $\nu=q_{S}$ and $T=\tau$ computed via a bifurcation analysis (as shown in Fig. 3). Thus, a unique MAW characterized by two parameters is selected in 1D by $\mathrm{BC}$ in Eq. (5) or in $2 \mathrm{D}$ by the two intrinsic frequencies of a meandering spiral. We conjecture, that the bifurcation diagram of superspirals in 2D can be predicted from the corresponding bifurcation diagram of the farfield MAWs provided the meandering period (that here corresponds to $\tau$ ) is known. Hence, the superspirals with saturated modulation should cease to exist in the saddlenode bifurcation of the associated MAW. If $\tau>T_{S N}$, the modulations grow monotonously and lead to appearance of space-time defects in $1 \mathrm{D}$, respectively topological defects in 2D (spiral breakup). In explicit 2D simulations, such behavior has already been observed in a homogeneous reaction-diffusion model for calcium waves, where simultaneous appearance of the Eckhaus instability and meandering leads to a breakup far away from the spiral core [21]. Future studies may address the possibility of MAWs in realistic reaction-diffusion systems which exhibit Eckhaus instabilities [20, 21]. A previous linear analysis of superspirals predicted that the meandering instability of a spiral with an Eckhaus unstable wavetrain in the far-field may produce superspirals with exponentially growing modulation, while in the standard case of a meandering spiral emitting a stable wave train exponential damping of the modulation should be observed [7]. Our nonlinear analysis introduces superspirals with a saturated modulation in the far-field as a third possibility, thus supporting the experimental ob- 
servation of such structures in the BZ reaction (Fig. 1 and [8]). We suggest an interpretation of the reported scenario damped superspiral - saturated superspiral - farfield breakup: the three phenomenologies simply correspond to the three mentioned regions of the bifurcation diagram for the MAWs. Notice that bifurcation diagrams similar to Fig. 3 (a) are found when $c_{1}$ or $c_{3}$ are varied at fixed $T$ 17, 18, 19].

Phase diagram. - Finally we determine the regions of the $c_{1}-c_{3}$ phase diagram [12 where the discussed phenomena may be found. The results are summarized in Fig. 4 where $\nu$ is fixed equal to $q_{S}\left(c_{1}, c_{3}\right)$ as given by Eq. (4). Saturated superspirals can appear only above the Eckhaus line for $q_{S}>q_{E}$. For small values of $c_{1}$, the MAWs bifurcate subcritically and are always unstable 22]. Fig. 4 also shows the line of absolute instability $\left(q_{S}=q_{A}\right)$ above which simple spirals break up giving rise to spatio-temporal chaos. Stable MAWs with $\nu=q_{S}$ do exist in the shaded region (for an extensive discussion on MAW stability see [18]). Below the thick dashed line in Fig. 4 no saddle-node bifurcation does occur and breakup is therefore prevented. Between the thick dashed and the thick dash-dotted lines in Fig. 4, bifurcation diagrams similar to Fig. 3(a) are found. In this region, all the three behaviors reported in Fig. 2 are possible, depending on the period of the forcing or meandering. Altogether, the results here presented link far-field breakup of meandering spirals to a saddle- node bifurcation. Thus, this route to spatio-temporal chaos can be distinguished from the previously reported scenario of far-field breakup, where spiral breakup has been explained by an absolute Eckhaus instability of the asymptotic far-field wavetrain [12, 13, 20, 23].

Conclusions. - We have studied the Doppler effect associated with the back-and-forth motion of a source emitting periodic nonlinear waves. Usually, the resulting modulation dies out by exponential damping as the waves move away from the source. If the emitted wavetrain is convectively Eckhaus unstable, the wave modulation can also saturate or grow exponentially far away from the source depending on the period of the backand-forth motion. These scenarios are fully determined by the bifurcation diagram of corresponding MAWs. Our results offer a consistent explanation of recent experimental results obtained in a chemical reaction [8]. Moreover, they may find application in future studies of other experimental systems exhibiting convective instabilities like hydrothermal waves 24. Periodic forcing of sources near the transition to chaotic or turbulent dynamics may be used to probe the existence and properties of modulated structures.

Acknowledgements - We acknowledge stimulating discussion with B. Sandstede and Q. Ouyang, in addition we would like to thank Q. Ouyang for the permission to report his experimental images in Fig. 1(a),(d).
* Electronic address: baer@mpipks-dresden.mpg.de

† Electronic address: torcini@ino.it

[1] M. C. Cross and P. C. Hohenberg, Rev. Mod. Phys. 65, 851 (1993); R. Kapral and K. Showalter (Eds.), Chemical waves and patterns (Kluwer, Dordrecht, 1994); J. Keener and J. Sneyd, Mathematical physiology (Springer, New York, 1998).

[2] P. S. Hagan, SIAM J. Appl. Math. 42, 762 (1982).

[3] Y. Kuramoto, Chemical oscillations, waves and turbulence (Springer, Berlin, 1984).

[4] I.S. Aranson and L. Kramer, Rev. Mod. Phys. 74, 99 (2002).

[5] A. S. Mikhailov, V. Davydov, and V. S. Zykov, Physica D 70, 1 (1994); M. Bär et al., J. Chem. Phys. 100, 1202 (1994); R. M. Mantel and D. Barkley, Phys. Rev. E 54, 4791 (1996).

[6] W. Jahnke, W. E. Skaggs, and A. T. Winfree, J. Phys. Chem. 93, 740 (1989); T. Plesser, S. C. Müller, and B. Hess, J. Phys. Chem. 94, 7501 (1990); G. S. Skinner and H. L. Swinney, Physica D 48, 1 (1991); D. Barkley, Phys. Rev. Lett. 68, 2090 (1992); G. Li et al., Phys. Rev. Lett. 77, 2105 (1996).

[7] B. Sandstede and A. Scheel, Phys. Rev. Lett. 86, 171 (2001).

[8] L. Q. Zhou, and Q. Ouyang, Phys. Rev. Lett. 85, 1650 (2000); J. Phys. Chem. A 105, 112 (2001).

[9] V. Perez-Munuzuri et al., Nature 353, 740 (1991).

[10] E. Bodenschatz, A. Weber, and L. Kramer, in Nonlinear Wave Processes in Excitable Media, edited by A. V.
Holden et al. (Plenum Press, New York, 1990).

[11] The function $\alpha$ is given by $\alpha\left(c_{1}, c_{3}\right)=$ $\sqrt{f\left(c_{1}, c_{3}\right) / g\left(c_{1}, c_{3}\right)}$ with $f\left(c_{1}, c_{3}\right)=3 c_{1}\left(8\left(c_{1}-\right.\right.$ $\left.\left.c_{3}\right)^{2}+9\left(1+c_{1} c_{3}\right)^{2}-4 c_{1} c_{3}\right)^{1 / 2}+c_{1}\left(5-9 c_{1} c_{3}\right)-4 c_{3}$ and $g\left(c_{1}, c_{3}\right)=4\left(-2 c_{3}+9 c_{1}^{3}+7 c_{1}\right)$.

[12] I. Aranson et al., Phys. Rev. A 46, 2992 (1992).

[13] S. M. Tobias and E. Knobloch, Phys. Rev. Lett. 80, 4811 (1998); Physica D 113, 43 (1998).

[14] D. Barkley, Phys. Rev. Lett. 72, 164 (1994).

[15] I. Aranson, L. Kramer, and A. Weber, Phys. Rev. Lett. 72, 2316 (1994).

[16] I. S. Aranson, L. Kramer, and A. Weber, in SpatioTemporal Patterns in Nonequilibrium Complex Systems, edited by P. E. Cladis and P. Palffy-Muhoray (AddisonWesley, Reading, 1995).

[17] L. Brusch et al., Phys. Rev. Lett. 85, 86 (2000); Physica D 160, 127 (2001).

[18] L. Brusch, A. Torcini, and M. Bär, Physica D 174, 152 (2003).

[19] M. van Hecke, Physica D 174, 134 (2003).

[20] M. Bär and M. Or-Guil, Phys. Rev. Lett. 82, 1160 (1999); B. Sandstede and A. Scheel, Phys. Rev. E 62, 7708 (2000).

[21] M. Falcke et al., Physica D 129, 236 (1999).

[22] B. Janiaud et al., Physica D 55, 269 (1992).

[23] Q. Ouyang and J. M. Flesselles, Nature 379, 143 (1996).

[24] N. Garnier, and A. Chiffaudel, Phys. Rev. Lett. 86, 75 (2001); N. Garnier, A. Chiffaudel, and F. Daviaud, Physica D 174, 30 (2003). 